\title{
Peculiar mean velocity profiles within a porous bed of an open channel
}

\author{
D. Pokrajac, C. Manes, and I. McEwan \\ Department of Engineering, University of Aberdeen, Aberdeen, AB24 3UE, United Kingdom
}

(Received 17 February 2007; accepted 7 August 2007; published online 26 September 2007)

\begin{abstract}
We present the velocity profile measured within a porous bed below rough turbulent open channel flow. Mean streamwise velocity has a minimum in the top pore, just below the bed surface, and then increases towards a constant value in the deeper pores. We argue that the velocity minimum in the top pore is due to enhanced turbulence generated within the external flow. This produces a flow regime similar to rough turbulent pipe flow, with very efficient momentum extraction. In the deeper pores turbulence intensities are damped so that momentum extraction is less efficient, allowing higher mean velocities to develop. (C) 2007 American Institute of Physics.
\end{abstract}

[DOI: $10.1063 / 1.2780193$ ]

For fluid flowing over and inside a rough permeable bed, the flow domain can be divided into a "free-stream" region above the bed and a subsurface region within it. Many studies have been carried out for the case of laminar subsurface flows. ${ }^{1-9}$ In contrast, due to experimental difficulties and highly demanding computational costs, laboratory investigations and numerical simulations of the turbulent flow inside permeable beds are very limited. ${ }^{10-13}$ This stimulated the authors to carry out a series of experiments designed to investigate turbulence properties at the interface between turbulent open channel flows and a porous bed. The internal geometry of the porous bed was chosen to allow velocity measurements within its voids and to maintain acceptable similarity with gravel beds of natural streams. For these purposes, the porous bed comprised uniform size spheres packed in cubic arrangement with each successive layer of spheres placed directly on top of the preceding one.

This Brief Communication focuses on mean streamwise velocity profiles measured within the bed. The profiles show a peculiar velocity minimum just one bead diameter below the bed surface. This was consistently found in all the experiments by two independent measurement techniques. This result is counterintuitive since one would expect mean velocities to decrease uniformly within the bed due to the weakening of the shearing effect induced by the free-stream flow. In the following text we provide an explanation for this unexpected result.

Experiments were conducted using an 11-m-long and 0.4-m-wide tilting hydraulic flume with a rectangular cross section. The permeable bed used in this study consisted of five layers of 12-mm-diam glass spheres packed in a cubic pattern. All experiments were carried out under uniform turbulent flow conditions. The main hydraulic parameters for the experiments are shown in Table I. Velocities were measured using an ultrasonic velocity profiler (UVP) and particle image velocimetry (PIV).

Subsurface velocities were measured by means of a 4-MHz Met-Flow UVP probe (Met-Flow SA 2000), which was inserted in a hole drilled in the bed and oriented along the flow, as shown in Fig. 1. In these experiments, the probe measured instantaneous velocities at 128 points within a length of about $95 \mathrm{~mm}$. Each measurement point corresponds to a disc-shaped sampling volume of 5-mm diam and 0.74-mm thickness. The sampling volumes did not overlap.

The streamwise velocity component, $u$, was measured at several heights within the bed by moving the UVP probe to four positions (named pores 1, 2, 3, and 4 in Fig. 1). The accuracy of velocities measured with UVP is about $0.8-1 \mathrm{~mm} / \mathrm{s}$ for all the experiments. Further details of the experimental technique and the validation of its applicability inside this porous bed can be found in Manes et al. ${ }^{14}$

PIV images were recorded by a KODAK ES1.0 digital cross-correlation camera using a double-pulsed Nd-Yag laser for illumination and VidPIV analysis software for determining the velocity vector field. The software applies a crosscorrelation algorithm over regions of the flow called interrogation areas. Average velocity in an interrogation area is calculated from the particle displacement found from the peaks in correlation function of the image gray-scale values. The subpixel position of these peaks is detected using three-point one-dimensional Gaussian fitting in two directions.

Free-stream velocity measurements were carried out with the laser placed in two central cross sections of the flume (Fig. 2): one over the tops of the balls ("Top") and another over their lateral connections ("Valley"). These two sections were chosen in order to capture the lateral heterogeneity of the flow within the roughness layer, where the flow is directly influenced by the individual roughness elements. Mean velocities were averaged over thin bed-parallel volumes, covering both top and valley sections in width, one bead diameter in length, and the size of the interrogation areas $(1.6 \mathrm{~mm})$ in height.

For one flow condition (20H400s) PIV was also used for measurement of velocities inside pores 1 and 2 (Fig. 2). In this case it was only possible to have a single measurement window with the illuminated plane through the valley section between the spheres. Furthermore, only the middle part was visible to the camera because the corners of the window were obstructed by the spheres.

PIV measurement accuracy was estimated to be $\sim 0.0025 \mathrm{~m} / \mathrm{s}$ for the free-stream experiments and $\sim 0.0018 \mathrm{~m} / \mathrm{s}$ for the measurements within pores 1 and 2 
TABLE I. Hydraulic conditions for the experiments; $H=$ flow depth measured from bead tops to free surface; $U_{b}=$ depth averaged velocity; $R$ $=B H /(B+2 H)=$ the hydraulic radius; $B=$ flume width; $K=$ bed permeability estimated by fitting Forchheimer's law through the velocities measured in the lowest pore; $\phi=$ bed porosity; $D=$ spheres diameter.

\begin{tabular}{|c|c|c|c|}
\hline \multirow[b]{2}{*}{ Parameter } & \multicolumn{3}{|c|}{ Experiment } \\
\hline & $20 \mathrm{H} 400 \mathrm{~s}$ & $21 \mathrm{H} 200 \mathrm{~s}$ & $42 \mathrm{H} 200 \mathrm{~s}$ \\
\hline$H(\mathrm{~mm})$ & 20 & 21 & 42 \\
\hline$S(\%)$ & 0.25 & 0.5 & 0.5 \\
\hline$U_{b}(\mathrm{~m} / \mathrm{s})$ & 0.254 & 0.369 & 0.535 \\
\hline $\operatorname{Re}=U_{b} H / \nu$ & 5588 & 8118 & 22470 \\
\hline $\operatorname{Re}_{\text {pore }}=\langle\bar{u}\rangle D / \nu$ & $373 \div 575$ & $507 \div 911$ & $408 \div 912$ \\
\hline$u_{*}=\sqrt{g S R}(\mathrm{~m} / \mathrm{s})$ & 0.0215 & 0.0312 & 0.0414 \\
\hline$K\left(\mathrm{~mm}^{2}\right)$ & & 1.9 & \\
\hline$\phi(\%)$ & & 48 & \\
\hline$D(\mathrm{~mm})$ & & 12 & \\
\hline
\end{tabular}

(Manes ${ }^{15}$ ). The size of the interrogation area was $\sim 1.6 \mathrm{~mm}$ for the free-stream measurements and $\sim 1.2 \mathrm{~mm}$ for pores 1 and 2 experiments.

Figure 3 shows the time-averaged streamwise velocities $\bar{u}$ obtained from UVP at different levels within the bed for experiment 20H400s (all other flow conditions show identical features). Time-averaged velocities generally increase in the narrower sections of the pores and decrease in the wider sections giving rise to the "wavy" profile in all pores.

However, there are notable differences between pore 1 and all other pores. The velocity in pore 1 is much smaller than in the other three pores. The "waviness" is much more pronounced, indicating that fluid inside pore 1 conforms to the pore geometry much more than in the other pores. Over a single wavelength velocity in pore 1 is symmetric, with approximately equal converging and diverging sections, whereas in the other pores there is asymmetry arising from a much shorter converging section in the flow.

In order to smooth the wavy spatial variability induced by the porous bed, mean velocities measured by the UVP in each pore were spatially averaged along their measurement length to obtain double-averaged (in time, then in space) velocities, denoted by $\langle\bar{u}\rangle$. The spatial averaging window covered the flow region along the measurement axis between 10 and $95 \mathrm{~mm}$ from the face of the transducer (seven sphere diameters, Fig. 3). The first $10 \mathrm{~mm}$ of each UVP measurement window was neglected because the velocities in this region were influenced by the presence of the transducer. Double-averaged velocity profiles are shown in Fig. 4.
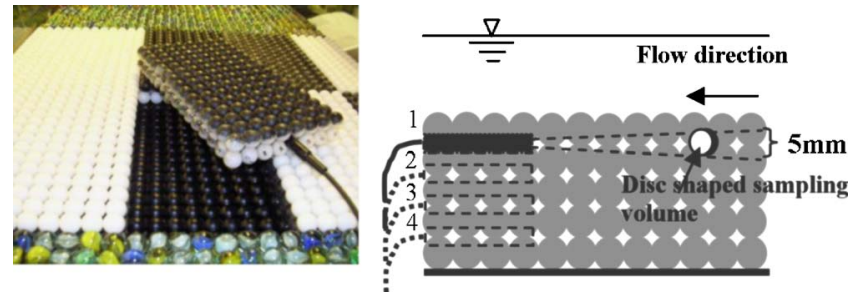

FIG. 1. (Color online) Positions of the UVP probe within the packed bed.

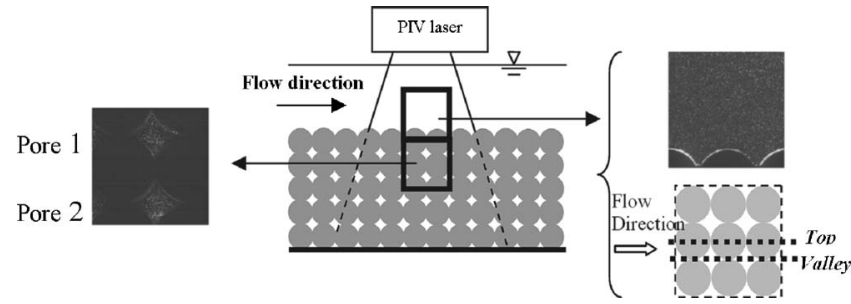

FIG. 2. Measurement windows for PIV experiments.

Intuitively, one would expect that mean velocities within the subsurface flow should gradually decrease within the bed due to the weakening of the shearing effect induced by the free-stream flow. Instead, mean velocities, $\langle\bar{u}\rangle$, in these experiments show the opposite behavior: they first decrease reaching a minimum in pore 1 from where they start to increase again creating an unexpected dip in the velocity profile [Figs. 4(a) and 4(b)]. This peculiar result was tested by using PIV to measure velocities in pores 1 and 2 . A detailed discussion of the comparison between pore velocities measured by UVP and PIV can be found in Manes et al. ${ }^{14}$ The PIV measurement confirmed that the velocities are indeed lower in pore 1 than in pore 2 [Fig. 4(c)]. This confirms that the dip is not a measurement artifact but rather the effect of physical mechanisms, which are discussed in the remaining text.

In gravity-driven steady and uniform open channel flows, mean longitudinal velocities within the bed are determined by the balance between the longitudinal component of gravity, the gradient of the fluid shear stress, and the drag the fluid exerts on the porous material, in this case uniform diameter spheres.

Mean velocity profiles inside the bed indicate that the effect of fluid shear induced by the free stream vanishes at roughly the height of the first pore. In fact, mean velocities below this level seem to depend only on bed slope and not on the free-stream hydraulic conditions (i.e., flow depth). This is further substantiated by the fact that Reynolds stress

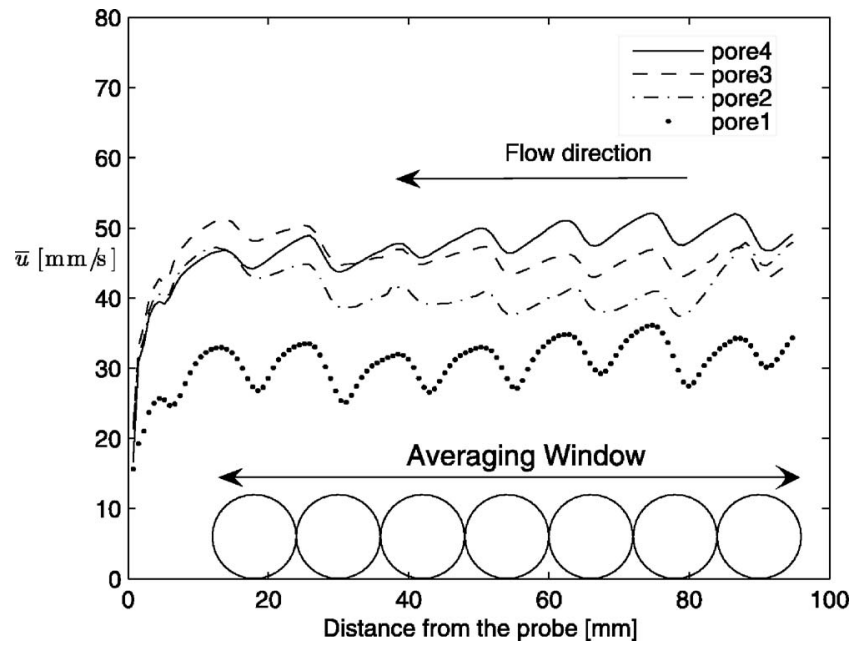

FIG. 3. Time-averaged velocities in experiment 20H400s. 


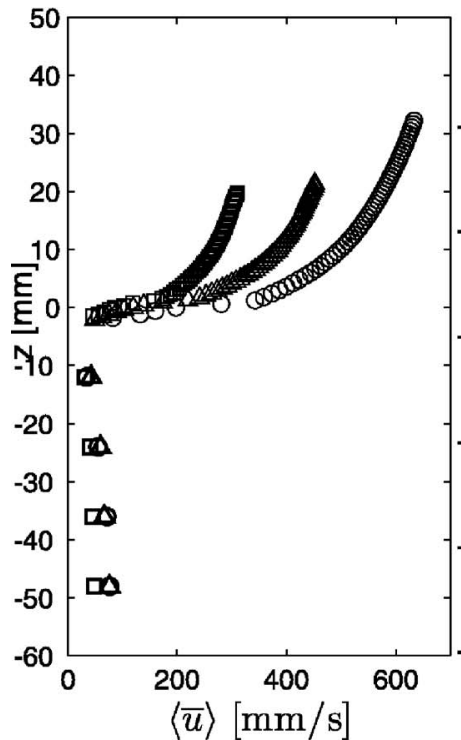

(a)

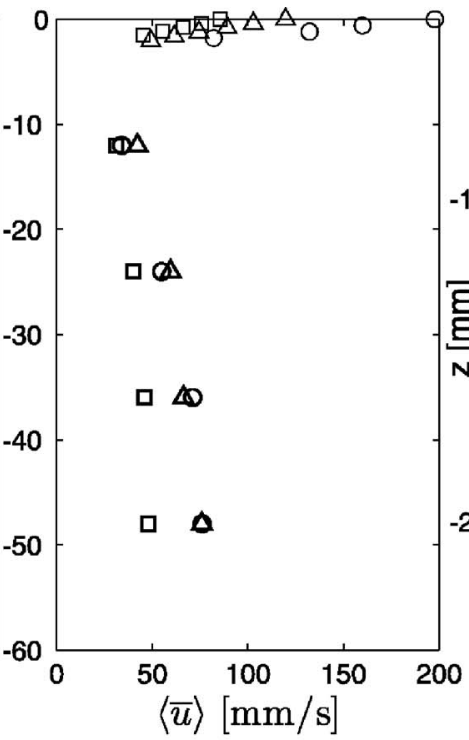

(b)

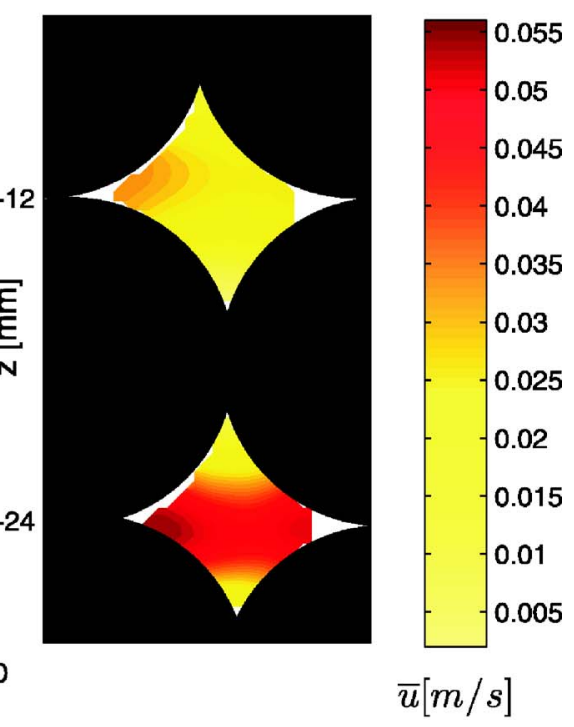

(c)

FIG. 4. (Color online) Mean velocity profiles in the (a) whole flow field and (b) focused within the porous bed. The origin for the $z$ coordinate is at the top of the uppermost sphere. The contour plot of $\bar{u}$ in (c) is obtained from PIV measurements within the bed in the 20H400s experiment. The black regions in the contour plot are the spheres forming pores 1 and 2 .

measured by PIV inside pores 1 and 2 (albeit only over a central plane) was very close to zero, as shown in Fig. 5(b).

Given that the free-stream shear penetration is negligible from pore 1 downwards, the momentum equation within the bed is characterized by a balance between gravity and drag forces. In other words, all the pores have an approximately equal momentum supply. For the velocity dip to exist under such conditions drag forces in the higher pores must be more efficient in extracting fluid momentum, i.e., the drag coeffi- cient must be higher, than in the lower pores. This increase in the drag coefficient is linked to the enhanced turbulence intensity close to the free stream. Figure 5(a) shows that the coefficient of variation of streamwise velocities (spatially averaged in the same manner as mean velocities) uniformly decreases within the bed. Higher coefficients of variation indicate higher turbulence intensities, which in turn, imply higher drag coefficients and ultimately lower mean velocities.

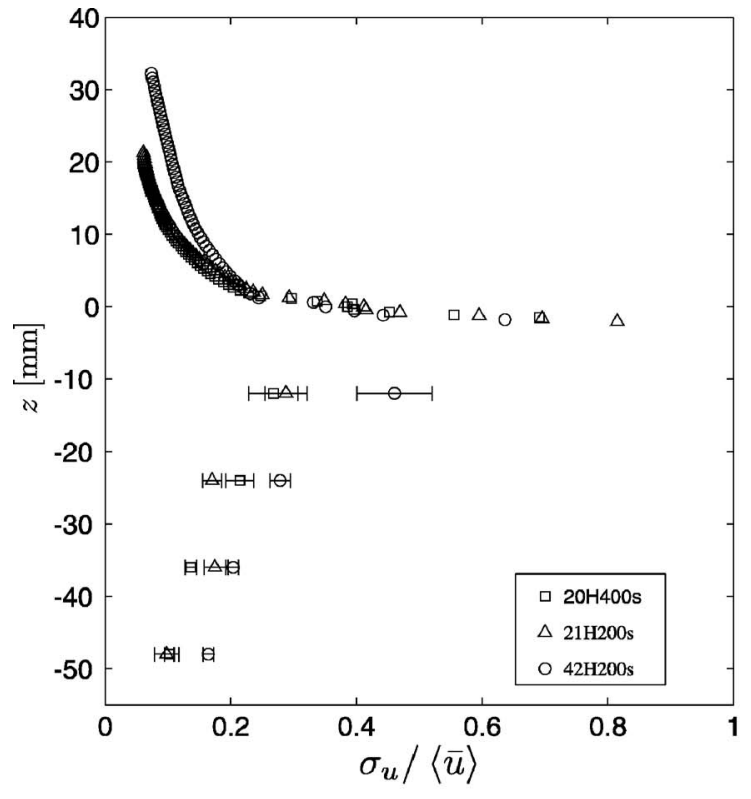

(a)

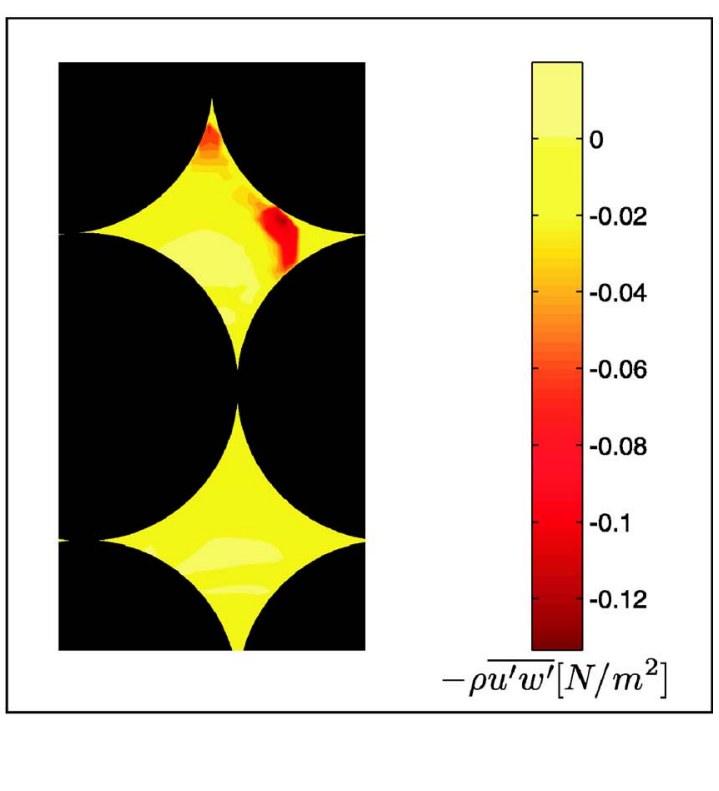

(b)

FIG. 5. (Color online) (a) Coefficient of variation $\sigma_{u} /\langle\bar{u}\rangle$, where $\sigma_{u}=\left\langle\sqrt{\overline{u^{\prime 2}}}\right\rangle$ is the rms of the longitudinal velocity fluctuations. A prime denotes time fluctuations. Spatial average (angle brackets) is applied along the same averaging window as for mean velocities (Fig. 3). Error bars show the standard deviation of the time-averaged statistics at the same phase along the wavelength of the periodic porous medium. (b) Reynolds stress in the top two pores in experiment the $20 \mathrm{H} 400 \mathrm{~s}$ experiment. Shear stress at roughness crest was $\tau=0.462 \mathrm{~N} / \mathrm{m}^{2}$. 
The phenomenon of the velocity dip can be explained if the longitudinal pores are understood as small and very rough pipes. Enhanced turbulence in the upper pore means that the fluid is mixed across the pore better, while in the lower pores the flow is concentrated in the middle of the pore, i.e., it separates from the spheres soon after the narrowest cross section. The spheres are closely packed so that the flow behind each individual sphere is intercepted by the next one, hence creating the so-called "sheltering effect." In such setup the region downstream from the separation point contains inactive, slowly spinning or completely stagnant fluid. When the separation point shifts downstream the stagnant fluid zone shrinks, exposing a bigger part of the sphere to faster fluid flow through the central section of the pore, hence increasing the drag coefficient. A similar phenomenon happens in rough pipes with uniform size roughness at the transition between smooth and rough pipe flow. The drag coefficient (friction factor) rapidly increases when the roughness elements start protruding through the top of the viscous sublayer. Thus, the top pore in our experiment can be described as hydraulically rough, while the lowest pores are hydraulically smooth. In terms of momentum balance, gravity is balanced mainly by form drag in the upper pores and mainly by viscous drag in the lower pores. Being less efficient in extracting momentum than form drag, viscous drag allows the fluid to develop higher velocities in the lower pores.

A specific feature of the flow inside the pores, and one which makes it different from conventional pipe flow, is that the turbulence responsible for changing the flow regime and hence enhancing drag originates in the external free-stream flow (Manes ${ }^{15}$ and Voellmer et al. ${ }^{13}$ ). Despite negligible net turbulent momentum transfer, externally generated turbulent kinetic energy penetrates into the top pore. This makes it possible to have rough turbulent flow at lower velocities and smooth turbulent flow at higher velocities. This is contrary to what happens in conventional pipe flow, where the turbulence is generated inside the pipe.

The velocity profile discussed in this Brief Communication is somewhat similar to that observed within plant canopies by Shaw, ${ }^{16}$ who found a velocity dip at the canopy crown and the secondary maximum at the bottom of the canopy. The shape of the canopy velocity profile is also due to a change in drag coefficients between the canopy crown and trunk regions, but caused by a change in geometry: branches and foliage of the crown provide more efficient blockage to air movement than the trunk. Our experimental results now show that the velocity dip can also occur in homogeneous porous materials.

In conclusion, we have presented a peculiar velocity profile for turbulent open channel flows inside a rough permeable bed, such that the minimum velocity occurs in the pore closest to the free stream. It was observed that the transport of free-stream momentum is negligible within the pores and hence the momentum exchange is characterized by the balance of the gravity and drag forces. Momentum extraction by drag is most efficient in the top pore, which is connected to the external flow. This is the result of the enhanced turbulence in that pore, which causes a flow regime similar to rough pipe flow, such that the form drag is the dominant mechanism of momentum extraction. Due to the turbulence intensity damping within the bed, the flow regime becomes closer to smooth pipe flow so that the drag coefficients decrease as the viscous drag becomes the more dominant part of the total drag. Being less efficient than form drag in extracting momentum from the flow, viscous drag allows higher mean velocities to develop in the lower pores. This is contrary to conventional pipe flow, where higher turbulence intensities occur at higher velocity, but still possible, because the turbulence that dictates the flow regime is generated within the external flow and not within the "pipe."

${ }^{1}$ G. S. Beavers and D. D. Joseph, "Boundary conditions at a naturally permeable wall," J. Fluid Mech. 30, 197 (1967).

${ }^{2}$ R. E. Larson and J. J. Higdon, "Microscopic flow near the surface of two-dimensional porous media. Part 1. Axial flow," J. Fluid Mech. 166, 449 (1986).

${ }^{3} \mathrm{~K}$. Vafai and R. Thiyagaraja, "Analysis of flow and heat transfer at the interface region of a porous medium," Int. J. Heat Mass Transfer 30, 1391 (1987).

${ }^{4}$ M. Sahraoui and M. Kaviany, "Slip and no-slip velocity boundary conditions at the interface of porous, plain media," Int. J. Heat Mass Transfer 35, 927 (1992).

${ }^{5}$ A. J. Ochoa-Tapia and S. Whitaker, "Momentum transfer at the boundary between a porous medium and a homogeneous fluid. I: Theoretical development," Int. J. Heat Mass Transfer 38, 2635 (1995).

${ }^{6}$ A. J. Ochoa-Tapia and S. Whitaker, "Momentum transfer at the boundary between a porous medium and a homogeneous fluid II: Comparison with experiment," Int. J. Heat Mass Transfer 38, 2647 (1995).

${ }^{7}$ C. Y. Choi and P. M. Waller, "Momentum transport mechanism for water flow over porous media," J. Environ. Eng. 123, 792 (1997).

${ }^{8}$ D. F. James, and A. M. Davis, "Flow at the interface of a model fibrous porous medium," J. Fluid Mech. 426, 47 (2001).

${ }^{9}$ U. Shavit, G. Bar-Yosef, and R. Rosenzweig, "Modified Brinkman equation for a free flow problem at the interface of porous surfaces: The Cantor-Taylor brush configuration case," Water Resour. Res. 38, 1320, DOI:10.1029/2001WR001142 (2002).

${ }^{10}$ J. F. Ruff and L. W. Gelhar, "Turbulent shear flow in porous boundary," J. Engrg. Mech. Div. 98, 975 (1972).

${ }^{11}$ W. P. Breugem, "The influence of wall permeability on laminar and turbulent flows. Theory and simulations," Ph.D. thesis, Delft University of Technology, 2005.

${ }^{12}$ Y. Shimizu, T. Tsujimoto, and H. Nakagawa, "Experiment and macroscopic modelling of flow in highly permeable porous medium under freesurface flow," J. Hydrosci. Hydr. Eng. 8, 69 (1990).

${ }^{13}$ S. Vollmer, R. Francisco de los Santos, H. Daebel, and G. Kuhn, "Microscale exchange processes between surface and subsurface water," J. Hydrol. 269, 3 (2002).

${ }^{14}$ C. Manes, D. Pokrajac, I. McEwan, V. Nikora, and L. J. Campbell, "A new application of UVP: velocity measurements within porous media," J. Hydraul. Eng. 132, 983 (2006).

${ }^{15}$ C. Manes, "Turbulent open channel flows over and within rough and permeable beds," Ph.D. thesis, University of Aberdeen, 2006.

${ }^{16}$ R. H. Shaw, "Secondary wind speed maxima inside plant canopies," J. Appl. Meteorol. 16, 514 (1977). 